\title{
Satisfacción laboral y Síndrome de Burnout en enfermeras del Servicio de Emergencia y Cuidados Críticos del Hospital San Bartolomé, 2010
}

\author{
Job satisfaction and burnout syndrome in nurses of the Emergency and Critical Care Hospital San \\ Bartolome, 2010
}

Yrma Nilda Broncano Vargas ${ }^{1}$

\begin{abstract}
RESUMEN
Objetivo: Establecer la relación entre la satisfacción laboral y la prevalencia de Síndrome de Burnout en enfermeras del Hospital San Bartolomé. Material y Métodos: Enfoque cuantitativo, descriptivo, prospectivo, transversal y correlacional. La muestra estuvo constituida por 45 enfermeras de los servicios de Emergencia y Cuidados críticos en el mes de agosto 2010. Se utilizó la ficha de datos sociolaborales, el cuestionario de satisfacción laboral S21/26 de J.L. Meliá y cuestionario para la evaluación del síndrome de quemarse por el trabajo (CESQT) de Pedro Gil-Monte. Los datos fueron procesados en el programa SPSS versión 18, el análisis inferencial se realizó mediante la prueba estadística chi cuadrado. Resultados: Existe Síndrome de Burnout alto en las enfermeras medianamente satisfechas $(50 \%)$; sin una asociación estadística significativa $(\mathrm{p}=0.914)$, rechazando la hipótesis nula. Las enfermeras que tienen Burnout bajo en la dimensión ilusión por el trabajo, tienen mayor satisfacción laboral $(75 \%)$, existiendo asociación estadística significativa $(\mathrm{p}=0.018<0.05)$. Quienes presentan Burnout alto y bajo en desgaste psíquico están medianamente satisfechas laboralmente, sin asociación estadística. Quienes tienen Burnout alto en la dimensión indolencia están medianamente satisfechas, sin asociación estadística y quienes presentan Burnout alto en la dimensión culpa están insatisfechas o medianamente satisfechas con su trabajo, sin una asociación estadística significativa. Conclusiones: No existe relación estadísticamente significativa entre satisfacción laboral y la prevalencia de Síndrome de Burnout en profesionales de enfermería, así como en las dimensiones desgaste psíquico, indolencia y culpa. Solo existe relación estadísticamente significativa entre satisfacción laboral y Síndrome de Burnout en la dimensión ilusión por el trabajo.
\end{abstract}

Palabras clave: Satisfacción laboral, Síndrome de Burnout, servicio de emergencia, servicios de cuidados críticos, enfermera profesional, enfermeras.

\begin{abstract}
Objective: To establish the relationship between job satisfaction and the prevalence of burnout syndrome in nurses in the Hospital San Bartolomé Lima Peru. Material and Methods: Quantitative approach, descriptive, prospective, cross-sectional and co-relational study. Population sample: 45 nurses of the Emergency and Critical Care Services, August 2010. The social and occupational data sheet was used for this research. Also, J.L. Melia's Job Satisfaction Questionnaire S21/26 and the Assessment Questionnaire burnout syndrome bound to work (CESQT) by Pedro Gil-Monte. The data were processed in SPSS version 18, statistical test for related: chi square. Results: There is high Burnout in moderately satisfied nurses (50\%); without a statistic significant association $(\mathrm{p}=0.914)$, rejecting the null hypothesis. The nurses who have low Burnout on the illusion dimension for work, have greater job satisfaction $(75 \%)$, existing statistical significant association $(\mathrm{p}=0.018<0.05)$. Those with high and low on Burnout psychological exhaustion are satisfied with work, without statistical association. Those with high burnout indolence dimension are fairly satisfied, without statistical association and those with high burnout on the guilt dimension are dissatisfied or moderately satisfied with their work, without a significant statistical association. Conclusions: There is no statistical significant relationship between job satisfaction and the burnout prevalence syndrome in nurses, as well as in their psychological exhaustion, indolence and guilt dimensions. There is a statistical meaningful relationship between job satisfaction and the burnout syndrome in their enthusiasm for the work dimension.
\end{abstract}

Keywords: Job satisfaction, burnout syndrome, emergency service, critical care services, Professional nurses.

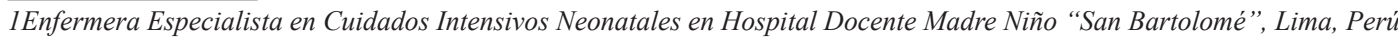




\section{INTRODUCCIÓN}

"La satisfacción laboral es un sentimiento de agrado que experimentan las personas, por el hecho de realizar un trabajo que les interesa en un ambiente que les permita estar a gusto, dentro de una empresa $\mathrm{u}$ organización que le resulte atractivo y por el que percibe una serie de compensaciones psico-socioeconómicas acordes a su expectativas" (Farfán, 2006).

Por otro lado, los estudios demuestran que la satisfacción laboral constituye un buen predictor de longevidad, adhesión a la institución, mejora la calidad de vida de los trabajadores, dentro y fuera del centro laboral, e influye favorablemente en el entorno social y familiar (Farfán, 2006; Chiang, Salazar y Núñez, 2007; Hernández, Quintana, Mederos y García, 2009).

Así también, existe evidencia que sostiene que un trabajador insatisfecho tiende a sufrir una serie de problemas de salud, tales como: cefaleas, estrés físico y mental, depresión mayor, accidentes cerebrovasculares, entre otros (Farfán, 2006).

Estas, a su vez, generan altas tasas de ausentismo, gastos médicos para el trabajador y costos para la institución, los cuales van en desmedro directo de la calidad de la atención de los usuarios externos.

Las enfermeras profesionales, que trabajan en el ámbito de recuperación de la salud, lo hacen diariamente en hospitales y clínicas enfrentando múltiples problemas que derivan tanto de la atención directa del paciente, como de la administración y coordinación del equipo de salud. Su misión de servicio las lleva a cuidar la salud física y mental de sus pacientes y entregar una atención de buena calidad. Para que esto ocurra, los profesionales deben estar bien y sentirse satisfechas en su centro de trabajo (Farfán, 2006; Chiang, Salazar y Núñez, 2007; Hernández, Quintana, Mederos y García, 2009).

Además, las enfermeras perciben, a veces, conflictos que se generan dentro de los equipos de trabajo, dificultades con sus jefes directos, con sus pares y con el personal a su cargo. El cansancio, las escasas posibilidades de capacitación, debido al sistema de turnos, y la escasez de personal traen a su vez fatiga y desmoralización, ellas perciben falta de reconocimiento hacia su grupo profesional.

Por otra parte, sus históricas bajas remuneraciones que a veces las obliga a desempeñar más de un trabajo, teniendo que asumir largas jornadas con el consiguiente desgaste que ello significa, agravan la situación. Asimismo, esta situación puede interferir en la vida familiar por los turnos rotativos, ya que a veces no coinciden con las rutinas normales de trabajo y estudios del resto de los miembros, por lo que la enfermera requiere de muchos esfuerzos para compatibilizar sus actividades con las de su familia.

Toda esta problemática trae, inevitablemente, consecuencias negativas y estrés en la vida personal y laboral (Ben, Bauzá, Cruz y Calvo, 2009).

En ese sentido, existen estudios en las últimas tres décadas que han identificado algunas enfermedades relacionadas al estrés laboral, como el denominado Síndrome de Burnout. Como consecuencia Dolan (1987) encontró que la insatisfacción laboral era un indicador fiable de "burnout" (Martínez-López y López - Solache, 2005).

El Síndrome de Burnout, término anglosajón o Síndrome de quemarse por el trabajo (SQT), para habla hispana, se relaciona con la exposición crónica a los estresores laborales, el trato diario con pacientes, originando la aparición de síntomas o molestias orgánicas (Martínez-López y López - Solache, 2005; Gil-monte, Marucco, 2008; Gil-monte, 2009). Se asocia con una disminución de rendimiento en el trabajo y con la baja satisfacción profesional. También llamado "Síndrome de estrés laboral", "Síndrome de desgaste profesional".

Fue introducido por primera vez por Freudemburger (1974), para describir las reacciones que presentan algunos profesionales como consecuencia de una exposición crónica a circunstancias laborales estresantes y al contacto directo con la gente (Sociedad Española de Cuidados Paliativos, 2008).

El modelo más conocido es de Maslach y Jackson (1981), con el instrumento Maslach Burnout Inventory (MBI), conceptualiza el Síndrome de Burnout como el síndrome tridimensional: agotamiento emocional, despersonalización y realización personal en el trabajo (Sociedad Española de Cuidados Paliativos, 2008).

Actualmente según Gil-monte (2006), el Síndrome de Burnout, o síndrome de quemarse por el trabajo (SQT), presenta cuatro dimensiones: ilusión por el trabajo, desgaste psíquico, indolencia y culpa (Olivares y Gil-monte, 2007).

Este síndrome ha requerido especial atención en el personal de salud y sobre todo en enfermeras, debido 
a que estos profesionales trabajan en contacto directo con personas enfermas, por lo que experimentan estrés físico, psíquico y emocional (Ozurk, 2006), además tienen alto grado de autoexigencia con baja tolerancia al fracaso, buscan la perfección absoluta, necesitan controlar todo o desarrollan un sentimiento de indispensabilidad laboral.

La prevalencia de esta patología varía de unos estudios a otros, encontrándose porcentajes del 30\% al $11.4 \%$ con respecto al personal que realiza algún trabajo de servicio, en países europeos, similares cifras en Latinoamérica.

Asimismo, diversas publicaciones nos señalan que las enfermeras que laboran en unidades de cuidados intensivos y de emergencia, tienen mayor incidencia al Síndrome de Burnout y se ven afectadas en algún momento de su vida laboral, en su salud física $\mathrm{y} / \mathrm{o}$ mental (Arce, Masgo, y Vásquez, 2006; Paredes, 2006)

Esto se relaciona con las características de trabajo que se desarrolla en estas unidades, donde el profesional de enfermería es altamente calificado, atiende a pacientes críticamente enfermos de grado IV que requieren valoración, monitoreo y cuidado permanente con asistencia intensiva; los pacientes suelen estar inestables, con compromiso multiorgánico severo, inconscientes pueden requerir sedación y/o relajación, ventilación asistida, con riesgo inminente de perder la vida o deteriorar su salud (Albaladejo, Ortega, Astasio, Calle y Domínguez, 2004).

En tal sentido, el estudio tuvo como objetivo establecer la relación entre la satisfacción laboral y la prevalencia de Síndrome de Burnout en profesionales de enfermería que laboran en los servicios de Emergencia y Cuidados Críticos del Hospital Nacional Docente Madre Niño San Bartolomé, formulando la hipótesis que existe relación entre la satisfacción laboral y la prevalencia de Síndrome de Burnout en profesionales de enfermería que laboran en los servicios de Emergencia y Cuidados Críticos del Hospital Nacional Docente Madre Niño San Bartolomé.

\section{MATERIAL Y MÉTODOS}

El estudio se realizó en el Hospital Nacional Docente Madre Niño San Bartolomé, Lima, Perú.
La población estuvo conformada por 45 enfermeras activas al mes de agosto del 2010 de los Servicios de Emergencia, Servicio de Cuidados Críticos del neonato, Servicio de Cuidados Críticos del niño y adolescente y Servicio de Cuidados Críticos de la mujer.

Fue de enfoque cuantitativo, descriptivo, prospectivo, transversal y correlacional. Se aplicó el instrumento, cumpliendo con los criterios de inclusión y exclusión. Se utilizó como técnica la encuesta y como instrumento el cuestionario autoadministrado. El cuestionario consta de tres instrumentos: el primero una ficha de datos sociolaborales elaborado, por la autora con el objetivo de identificar y caracterizar al personal de salud.

El segundo instrumento fue el "Cuestionario de Satisfacción Laboral S21/26” de José Meliá en 1998, ofrece una medida sencilla de bajo costo, con un nivel de fiabilidad y validez propio de los cuestionarios con gran número de ítemes. Este cuestionario se caracteriza por un formato dicotómico de respuesta (verdadero o falso) de veinticinco ítemes que lo hace muy indicado para el trabajo profesional.

El tercer instrumento fue el "Cuestionario para la Evaluación del Síndrome de Quemarse por el Trabajo" (CESQT) de Pedro Gil-monte el 2006, instrumento de medición de Síndrome de Burnout, proporcionado por el mismo autor para esta investigación, instrumento validado y fiable para evaluar el SQT en profesionales que trabajan brindando atención a personas con discapacidad, fue aplicado en muestras de España, Portugal, Costa Rica, México, Chile, Brasil, Colombia, Argentina y otros países latinoamericanos, asimismo, por primera vez en Perú. El cuestionario está formado de veinte ítemes que se evalúan mediante una escala Likert, con cinco opciones de respuesta.

Los datos fueron procesados en el programa SPSS versión 18, prueba estadística para establecer la relación fue el chi cuadrado, con un nivel de confianza del $95 \%$. El análisis de los datos se realizó en dos momentos. Un primer momento descriptivo; donde se presentó tablas de todas las variables y un segundo nivel de análisis, para buscar la relación entre las variables. 


\section{RESULTADOS}

\section{Tabla 1}

Satisfacción laboral del profesional de enfermería que labora en los servicios de Emergencia y Cuidados Críticos del Hospital Nacional Docente Madre Niño San Bartolomé, 2010.

\begin{tabular}{lll}
\hline Satisfacción laboral & N. ${ }^{\circ}$ & $\%$ \\
\hline Satisfecho & 7 & 15.6 \\
Medianamente satisfecho & 29 & 64.4 \\
Insatisfecho & 9 & 20 \\
Total & 45 & 100 \\
\hline
\end{tabular}

En la tabla 1 se evidencia que, en los servicios de Emergencia y Cuidados Críticos del Hospital Nacional Docente Madre Niño San Bartolomé, el $64.4 \%$ de enfermeras se encuentra con una satisfacción laboral media, el $20 \%$ se encuentra insatisfecho con el trabajo actual que tiene, y solo el $15.6 \%$ está satisfecho laboralmente en la institución.

Tabla 2

Síndrome de Burnout del profesional de enfermería que labora en los Servicios de Emergencia y Cuidados Críticos del Hospital Nacional Docente Madre Niño San Bartolomé, agosto 2010.

\begin{tabular}{lll}
\hline Síndrome de Burnout & $\mathrm{N} .^{\circ}$ & $\%$ \\
\hline Bajo & 5 & 11.1 \\
Intermedio & 34 & 75.6 \\
Alto & 6 & 13.3 \\
Total & 45 & 100 \\
\hline
\end{tabular}

Se aprecia, en la tabla 2, que en los Servicios de Emergencia y Cuidados Críticos, el $75.6 \%$ de enfermeras padece de un nivel intermedio de
Síndrome de Burnout, el $11.1 \%$ de enfermeras padece de un nivel bajo de Burnout y un $13.3 \%$ de ellas padece de un nivel alto de Síndrome de Burnout.

\section{Tabla 3}

Relación entre la satisfacción laboral y la prevalencia de Síndrome de Burnout en profesionales de enfermería que laboran en los Servicios de Emergencia y Cuidados Críticos del Hospital Nacional Docente Madre Niño San Bartolomé, agosto 2010.

\begin{tabular}{|c|c|c|c|c|c|c|c|c|}
\hline \multirow{3}{*}{ Satisfacción laboral } & \multicolumn{8}{|c|}{ Síndrome de Burnout } \\
\hline & \multicolumn{2}{|c|}{ Bajo } & \multicolumn{2}{|c|}{ Intermedio } & \multicolumn{2}{|c|}{ Alto } & \multicolumn{2}{|c|}{ Total } \\
\hline & N. ${ }^{\circ}$ & $\%$ & N. ${ }^{\circ}$ & $\%$ & N. ${ }^{\circ}$ & $\%$ & N. ${ }^{\circ}$ & $\%$ \\
\hline Insatisfecho & 1 & 20 & 6 & 17.7 & 2 & 33.3 & 9 & 20 \\
\hline Medianamente satisfecho & 3 & 60 & 23 & 67.6 & 3 & 50 & 29 & 64.4 \\
\hline Satisfecho & 1 & 20 & 5 & 14.7 & 1 & 16.7 & 7 & 15.6 \\
\hline Total & 5 & 100 & 34 & 100 & 6 & 100 & 45 & 100 \\
\hline
\end{tabular}

En la tabla 3, con respecto a la relación entre la satisfacción laboral y la prevalencia de Síndrome de Burnout en profesionales de enfermería que laboran en los Servicios de Emergencia y Cuidados Críticos, encontramos que hay mayor frecuencia de Burnout alto en el personal medianamente satisfecho (50\%); sin una asociación estadística significativa $(\mathrm{p}=0.914)$, rechazando la hipótesis. 
Satisfacción laboral y Síndrome de Burnout en enfermeras del Servicio de Emergencia y Cuidados Críticos del Hospital San Bartolomé, 2010

Tabla 4

Relación entre la satisfacción laboral y el Síndrome de Burnout en su dimensión Ilusión por el trabajo en profesionales de enfermería que laboran en los servicios de Emergencia y Cuidados Críticos del Hospital Nacional Docente Madre Niño San Bartolomé, agosto 2010.

\begin{tabular}{lcccccccc}
\hline & \multicolumn{8}{c}{ Burnout: Dimensión ilusión por el trabajo } \\
\cline { 2 - 9 } Satisfacción laboral & \multicolumn{2}{c}{ Bajo } & \multicolumn{2}{c}{ Intermedio } & Alto & \multicolumn{3}{c}{ Total } \\
& N. & $\%$ & N. ${ }^{\circ}$ & $\%$ & N. ${ }^{\circ}$ & $\%$ & N. ${ }^{\circ}$ & $\%$ \\
\hline Insatisfecho & 1 & 14.3 & 5 & 19.2 & 3 & 25 & 9 & 20.0 \\
$\begin{array}{l}\text { Medianamente } \\
\text { satisfecho }\end{array}$ & 2 & 28.6 & 18 & 69.2 & 9 & 75 & 29 & 64.4 \\
Satisfecho & 4 & 57.1 & 3 & 11.6 & 0 & 0 & 7 & 15.6 \\
Total & 7 & 100 & 26 & 100 & 12 & 100 & 45 & 100 \\
\hline
\end{tabular}

$\mathrm{X}^{2}=11.89, \quad p=0.018$

En cuanto a la relación entre la satisfacción laboral y la prevalencia de Síndrome de Burnout, según la dimensión Ilusión por el trabajo en profesionales de enfermería que laboran en los servicios de Emergencia y Cuidados Críticos, encontramos que hay una mayor frecuencia de Burnout alto en el personal medianamente satisfecho (75\%); con asociación estadística significativa ( $\mathrm{p}=0.018$ ), además, el personal de enfermería con Burnout bajo tiene mayor frecuencia de satisfacción laboral (57.1\%).

\section{Tabla 5}

Relación entre la satisfacción laboral y el Sindrome de Burnout en su dimensión Desgaste psíquico en profesionales de enfermería que laboran en los Servicios de Emergencia y Cuidados Críticos del Hospital Nacional Docente Madre Niño San Bartolomé, agosto 2010.

\begin{tabular}{|c|c|c|c|c|c|c|c|c|}
\hline \multirow{3}{*}{ Satisfacción laboral } & \multicolumn{8}{|c|}{ Burnout: dimensión desgaste psíquico } \\
\hline & \multicolumn{2}{|c|}{ Bajo } & \multicolumn{2}{|c|}{ Intermedio } & \multicolumn{2}{|c|}{ Alto } & \multicolumn{2}{|c|}{ Total } \\
\hline & N. ${ }^{\circ}$ & $\%$ & N. ${ }^{\circ}$ & $\%$ & N. ${ }^{\circ}$ & $\%$ & N. ${ }^{\circ}$ & $\%$ \\
\hline Insatisfecho & 4 & 44.4 & 5 & 17.2 & 0 & 0 & 9 & 20 \\
\hline $\begin{array}{l}\text { Medianamente } \\
\text { satisfecho }\end{array}$ & 5 & 55.6 & 19 & 65.6 & 5 & 71.4 & 29 & 64.4 \\
\hline Satisfecho & 0 & 0 & 5 & 17.2 & 2 & 28.6 & 7 & 15.6 \\
\hline Total & 9 & 100 & 29 & 100 & 7 & 100 & 45 & 100 \\
\hline
\end{tabular}

$\mathrm{X}^{2}=6.583, \quad p=0.160$

En cuanto a la relación entre la satisfacción laboral y la prevalencia de síndrome de Burnout, según la dimensión desgaste psíquico en profesionales de enfermería que laboran en los servicios de Emergencia y Cuidados Críticos, encontramos que hay una mayor frecuencia de Burnout alto en el personal medianamente satisfecho (71.4\%); sin una asociación estadística significativa $(\mathrm{P}=0.160)$, además el personal de enfermería con Burnout bajo tiene mayor frecuencia de satisfacción laboral media $(55.6 \%)$. 
Tabla 6

Relación entre la satisfacción laboral y el Síndrome de Burnout en su dimensión Indolencia en profesionales de enfermería que laboran en los Servicios de Emergencia y Cuidados Críticos del Hospital Nacional Docente Madre Niño San Bartolomé, agosto 2010.

\begin{tabular}{lllllllll}
\hline \multirow{2}{*}{ Satisfacción Laboral } & \multicolumn{8}{c}{ Burnout: dimensión indolencia } \\
\cline { 2 - 10 } & \multicolumn{2}{c}{ N. $^{\circ}$} & $\%$ & \multicolumn{1}{c}{ Intermedio } & Alto & \multicolumn{3}{c}{ Total } \\
& 3 & 42.8 & 4 & 13.3 & 2 & 25 & 9 & 20 \\
\hline Insatisfecho & 2 & 28.6 & 22 & 73.4 & 5 & 62.5 & 29 & 64.4 \\
$\begin{array}{l}\text { Medianamente } \\
\text { satisfecho }\end{array}$ & 2 & & & & & & & \\
Satisfecho & 2 & 28.6 & 4 & 13.3 & 1 & 12.5 & 7 & 15.6 \\
Total & 7 & 100 & 30 & 100 & 8 & 100 & 45 & 100 \\
\hline
\end{tabular}

$\mathrm{X}^{2}=5.271, \quad p=0.261$

En cuanto a la relación entre la satisfacción laboral y la prevalencia de Síndrome de Burnout según la dimensión indolencia en profesionales de enfermería, que laboran en los Servicios de Emergencia y Cuidados Críticos, encontramos que hay una mayor frecuencia de Burnout alto en el personal medianamente satisfecho $(62.5 \%)$; sin una asociación estadística significativa $(\mathrm{p}=0.261)$, además el personal de enfermería con Burnout bajo tiene mayor frecuencia de insatisfacción laboral (42.8\%).

Tabla 7

Relación entre la satisfacción laboral y el Sindrome de Burnout en su dimensión Culpa en profesionales de enfermería que laboran en los Servicios de Emergencia y Cuidados Críticos del Hospital Nacional Docente Madre Niño San Bartolomé, agosto 2010.

\begin{tabular}{|c|c|c|c|c|c|c|c|c|}
\hline \multirow{3}{*}{ Satisfacción Laboral } & \multicolumn{8}{|c|}{ Burnout: dimensión culpa } \\
\hline & \multicolumn{2}{|c|}{ Bajo } & \multicolumn{2}{|c|}{ Intermedio } & \multicolumn{2}{|c|}{ Alto } & \multicolumn{2}{|c|}{ Total } \\
\hline & N. ${ }^{\circ}$ & $\%$ & N. ${ }^{\circ}$ & $\%$ & N. ${ }^{\circ}$ & $\%$ & N. ${ }^{\circ}$ & $\%$ \\
\hline Insatisfecho & 1 & 12.5 & 5 & 16.7 & 3 & 42.9 & 9 & 20 \\
\hline $\begin{array}{l}\text { Medianamente } \\
\text { satisfecho }\end{array}$ & 6 & 75 & 20 & 66.6 & 3 & 42.9 & 29 & 64.4 \\
\hline Satisfecho & 1 & 12.5 & 5 & 16.7 & 1 & 14.2 & 7 & 15.6 \\
\hline Total & 8 & 100 & 30 & 100 & 7 & 100 & 45 & 100 \\
\hline
\end{tabular}

En cuanto a la relación entre la satisfacción laboral y la prevalencia de Síndrome de Burnout, según la dimensión Culpa en profesionales de enfermería que laboran en los Servicios de Emergencia y Cuidados Críticos; encontramos que hay mayor frecuencia

\section{DISCUSIÓN}

Las enfermeras de los servicios de Emergencia y Cuidados Críticos del Hospital Nacional Docente Madre Niño San Bartolomé presentan una satisfacción laboral media, coincidiendo con autores como (Colunga, Domínguez, González, Del Castillo, de Burnout alto en el personal insatisfecho y medianamente satisfecho (85.8\%); sin una asociación estadística significativa $(\mathrm{P}=0.563)$, además el personal de enfermería con Burnout bajo tiene mayor frecuencia de satisfacción laboral media (75\%).

y Solorio, 2008) quienes en su estudio, "Satisfacción laboral y Burnout en enfermeras de tercer nivel de atención en Guadalajara", reportan que la satisfacción laboral frecuente fue moderada; en similitud con (Ríos y Godoy, 2008) en su investigación "Satisfacción Laboral en un colectivo de enfermería de Urgencias y (Ríos- Risquez y Godoy- Fernández, 2008) en su 
trabajo "Relación entre satisfacción laboral y salud general percibida en profesionales de enfermería de urgencias" de España, quienes concluyen que el grado de satisfacción laboral en las enfermeras es medio, resultados que a su vez demuestra que los profesionales presentan vulnerabilidad en su salud psíquica, al respecto (Cantera, 2008) refiere que la insatisfacción influye decisivamente en el estado anímico de la persona y su conducta; como factor de efecto la insatisfacción actúa como detonante de alteraciones psicosomáticas y puede llegar a producir tensión e incluso enfermedad, correlaciona positivamente con el estado de ansiedad, trastornos gastrointestinales, estress y alteraciones diversas, resultando en consecuencia un riesgo en la calidad de atención al paciente, según (Marucco y Gil-montes, 2007), en el caso del personal de salud, a mayor satisfacción laboral existente generalmente mejor calidad de atención, lo que finalmente repercutirá en la pronta mejoría del paciente, coincidiendo con (Hernández, Quintana, Mederos, Guedes y García, 2009), la misión de servicio de las enfermeras las lleva a cuidar la salud física y mental de sus pacientes y entregar una atención de buena calidad, para que esto ocurra, las profesionales deben estar bien y sentirse satisfechas en su trabajo.

Con respecto al Síndrome de Burnout, nuestro estudio muestra que las enfermeras en su mayoría presentan un nivel medio de Burnout y un mínimo de ellas padece de un nivel alto, nuestros datos son similares a los resultados de (Morales, 2006), en su estudio "Nivel de estrés laboral de las enfermeras que laboran en las áreas críticas del Instituto Nacional de Salud del Niño", quienes reportan que el mayor porcentaje de enfermeras presenta un nivel de estrés laboral moderado, a diferencia de (Arce, Masgo, Vásquez, 2006), quienes en su estudio "Síndrome de Burnout en profesionales de enfermería de la Unidad de Cuidados Críticos del Hospital Guillermo Almenara Irigoyen", 2006 concluyen que las enfermeras de cuidados intensivos, aunque no tienen Síndrome de Burnout, la mayoría de ellas se encuentra con tendencia a desarrollar este síndrome; según (Martínez - López y López-Solache, 2005), el ejercicio de la profesión de enfermería lleva consigo gran responsabilidad sobre la vida, la salud y el cuidado de otras personas. La enfermera que labora en las unidades críticas requiere de un gran esfuerzo mental y físico; aumenta el nivel de carga del trabajo ante las diversas situaciones difíciles que enfrenta a diario, principalmente en el sufrimiento humano y la muerte.

Asimismo, el estudio realizado revela que la dimensión predominante del Síndrome de Burnout en las enfermeras de estos servicios es la indolencia seguida por igual en desgaste psíquico, desilusión por el trabajo y culpa, coincidiendo parcialmente con (Tuesca-Molina, Iguarán, Vargas, y Vergara, 2006), donde los resultados obtenidos, en las diferentes subescalas del Burnout, son en primer lugar la despersonalización, luego cansancio emocional $\mathrm{y}$, en tercer lugar, la baja realización personal, no considerando la culpa como dimensión. A diferencia de lo que refiere (Tello, 2010), por igual la relevancia de agotamiento emocional y despersonalización; y, en último lugar, la baja realización personal.

Así también, el modelo teórico que subyace al CESQT desarrollado por (Olivares y Gil-Monte, 2005) considera, en este modelo, que el deterioro cognitivo (bajas puntuaciones en Ilusión por el trabajo) y afectivo (altas puntuaciones en desgaste psíquico) aparecen, en un primer momento, como respuesta a las fuentes de estrés laboral crónico y, con posterioridad, los individuos desarrollarán actitudes negativas hacia las personas que atienden en su trabajo (altos niveles de indolencia). La aparición de los sentimientos de culpa es posterior a estos síntomas, no presentándose en todos los individuos.

Al realizar el cruce de variables de satisfacción laboral y síndrome de Burnout, en enfermeros que laboran en los servicios de Emergencia y Cuidados Críticos del Hospital Nacional Docente Madre Niño San Bartolomé, nuestro estudio refiere que existe Burnout alto en el personal medianamente satisfecho, sin una asociación estadística significativa, estableciendo que no existe relación entre la baja satisfacción laboral y el Síndrome de Burnout. Asimismo (Colunga, Domínguez, González, Del Castillo y Solorio, 2008), en su trabajo sobre "Satisfacción laboral y Burnout en enfermeras de tercer nivel de atención en Guadalajara", concluyen que no encontraron asociación significativa entre la baja satisfacción laboral y la presencia de Burnout, dato que coincide con nuestro estudio, a diferencia de (Albaladejo, Villanueva, Ortega, Astasio, Calle y Domínguez, 2004), quienes en su estudio "Síndrome de Burnout en el personal de enfermería de un hospital de Madrid" mencionan que a menor grado de satisfacción laboral más altas son las puntuaciones en las 4 escalas del Síndrome de Burnout, concluyendo que el perfil del sujeto afecto de Burnout corresponde a uno, con alto nivel de insatisfacción con la gestión de su centro de trabajo; con lo cual nuestro estudio difiere. Según (Santamaría, 2010), uno de los grupos más afectados por el estrés en el trabajo diario lo constituyen los profesionales de enfermería. Existen factores de riesgo como las condiciones laborales que generan muchos efectos negativos sobre el trabajador 
entre otros, conflicto entre los miembros del equipo, la carencia de confianza y autonomía, el exceso de trabajo, conflictos de autoridad".

Al respecto, el Dr. Horacio Cairo según (Thomaé, Alaya, Sphan y Stortli, 2006) propone puntos claves en la prevención del Burnout: Un proceso personal de adaptación de expectativa a la realidad cotidiana. Formación en las emociones, equilibrio de áreas vitales: familia, amigos, aficiones, descansos y trabajo. Fomento de buena atmósfera de equipo: espacios comunes, objetivos comunes. Limitar a un máximo la agenda asistencial. Tiempo adecuado por pacientes. Minimizar la burocracia con mejora en la disponibilidad de recursos materiales de capacitación, mejorando la remuneración. Formación continuada dentro de la jornada laboral. Coordinación con los colegas que ejercen la misma especialidad, espacios comunes, objetos compartidos. Diálogo efectivo con los jefes y gerencias.

Por otro lado, (Thomaé, Alaya, Sphan y Stortli, 2006) refiere: El Síndrome de Burnout puede ser bien manejado por todos aquellos que trabajan en el ámbito de la salud si fuesen informados y prevenidos sobre el mismo, también enseñándoles cómo tolerar o deshacerse de todas aquellas situaciones que generen emociones encontradas con uno mismo o con las diversas personas (directivos, colegas, familiares, amigos, etc.) que tratan diariamente y contamos con suficientes armas terapéuticas para tratarlo. Además, (González, Oramas, Alvarez y Oliva, 2006) enfatiza que el Síndrome del Burnout en el enfermero, genera alteraciones en el bienestar de la población atendida y opaca el reconocimiento social de estos profesionales de la salud, por lo cual tratar y luchar contra este síndrome significa para Enfermería luchar a favor de un desarrollo y reconocimiento de la profesión.

Así también resulta relevante que los profesionales que experimentan Burnout bajo en la dimensión ilusión por el trabajo tiene mayor satisfacción laboral, existiendo relación con asociación estadística significativa. Al realizar la revisión bibliográfica, no encontramos estudios con resultados similares. Con respecto a la ilusión por el trabajo o realización personal para Maslach, (Gil - monte, García Juesas, Núñez, Carretero y Roldan, 2006) refieren que la ilusión por el trabajo es el deseo del individuo de alcanzar las metas laborales, porque supone una fuente de placer personal. El enfermero percibe su trabajo atractivo y alcanzar las metas profesionales es la fuente de realización personal. Esta dimensión está formulada de manera inversa positiva: altos niveles de Burnout indican bajas puntuaciones de ilusión y bajos niveles de Burnout indican altas puntuaciones de ilusión, respaldando nuestros resultados donde los enfermeros que presentan bajo Burnout en esta dimensión, reflejan alta ilusión por el trabajo $\mathrm{y}$ presentan adecuada satisfacción laboral.

Así también, los enfermeros de unidades críticas y emergencia de nuestro estudio que presentan Burnout alto en desgaste psíquico, así como Burnout bajo en desgaste psíquico se encuentra medianamente satisfechos con su actividad laboral, sin una asociación estadística significativa. Después de realizar una revisión bibliográfica no se encuentra investigación con resultados similares, sin embargo, resulta relevante rescatar que según (Milachay, 2006), en "Factores personales y organizacionales relacionadas con el síndrome de Burnout del profesional de enfermería del Hospital Rene Toche Groppo. EsSalud Chincha", existe una elevada prevalencia del síndrome de Burnout, siendo mayor en la dimensión agotamiento emocional. Sobre el agotamiento emocional o desgaste psíquico, (Ortega y López, 2004) menciona que es la sensación creciente de agotamiento en el trabajo, "de no poder dar más de sí", debido a que se tiene que tratar a diario con personas que presentan o causan problemas.

Además (Colunga, Domínguez, González, Del Castillo y Solorio, 2008) refiere que este síndrome, ha requerido especial atención en el personal de salud, debido a que estos profesionales trabajan en contacto directo con personas enfermas, por lo que experimentan estrés físico, psíquico y emocional, además las enfermeras de unidades críticas presentan alto grado de autoexigencia con baja tolerancia al fracaso, buscan la perfección absoluta, necesitan controlar todo o desarrollan un sentimiento de indispensabilidad laboral. Asimismo, refiere (González, Oramas, Alvarez y Oliva, 2006), que el agotamiento emocional se traduce como pérdida de los recursos emocionales del enfermero ante sus pacientes; el profesional se siente cansado con toda una serie de manifestaciones orgánicas como son las psicosomáticas, entre las que se encuentran: cefalea, úlceras, alteraciones del sueño y disfunciones sexuales, que hacen que no pueda ser capaz de pensar en la magnitud de los problemas de los pacientes, ni en su solución.

Además, nuestro estudio revela Burnout alto en la dimensión indolencia en el personal de enfermería medianamente satisfecho, sin una asociación estadística significativa, también el personal de enfermería con Burnout bajo en la dimensión indolencia se encuentra con insatisfacción laboral. No encontramos estudios similares o referentes. Al respecto sobre indolencia o despersonalización (Gil- 
monte, García-Juesas, Núñez, Carretero, Roldán y Caro, 2006) comentan que es la presencia de actitudes negativas de indiferencia y cinismo hacia los clientes o pacientes. Los enfermeros que tienen alta puntuación, en esta dimensión, muestran insensibilidad y no se conmueven ante los problemas de las personas hacia las que trabajan. Según (Gonzales, Oramas, Alvarez y Oliva, 2006), la despersonalización es el resultado de la aparición de manifestaciones emocionales; por ejemplo, el distanciamiento afectivo como forma de protección del yo, actitud cínica, impaciencia e irritabilidad, lo que engendra una actitud, en la que se pierde el respeto al paciente; se es injusto en su trato, no se hace el bien, se le hace daño, pues ante el deterioro de su salud, el enfermo se sumerge en la angustia y ve como único consuelo las esperanzas que el profesional sea capaz de darle. Además, el personal afectado deja de tener en cuenta las armas de sensibilidad y humanismo con las que debe respetar la capacidad de decisión, además de la integridad física y psíquica del paciente, por lo que se genera un distanciamiento afectivo que puede engendrar una actitud que corroe los elementos éticos esenciales. Por otro lado, (Ortega y López, 2004) refiere que se crea un desarrollo de sentimientos y actitudes negativas, respuestas frías e impersonales, también hacia los compañeros de trabajo.

Los enfermeros de las unidades críticas y de emergencia que presentan Burnout alto en la dimensión culpa están insatisfechos o medianamente satisfechos con su trabajo, sin una asociación estadística significativa. Al respecto (Olivares y Gilmonte, 2007) refiere que los sentimientos de culpa son síntomas característicos de los individuos que desarrollan el síndrome. Aparecen los sentimientos de culpa por el comportamiento y las actitudes negativas desarrolladas en el trabajo, en especial hacia las personas con la que se establecen relaciones laborales.

Con respecto a algunas características sociolaborales, la mayoría de enfermeras en las unidades críticas y emergencia del Hospital Nacional
Docente Madre Niño San Bartolomé son de sexo femenino, adultas maduras entre 30 a 39 años de edad, casadas o convivientes, tienen carga familiar de 2 hijos, son nombradas, laboran en el hospital entre 10 a 19 años y de 2 a 4 en la unidad crítica, coincidiendo con las características de las enfermeras de áreas críticas del Instituto Nacional de Salud del Niño como señala (Morales, 2006), son adultas maduras de 36 a 44 años, casadas, tienen un tiempo de servicio de cuatro años a más y son nombradas; al respecto refiere (Arias, 2008), la gran cantidad de obligaciones del personal de enfermería en el ambiente laboral, así como las obligaciones familiares, mantenimiento de la casa, hijos y deberes como pareja, entre otras, influyen en el aumento de los niveles del estrés.

Así también, resulta significativo para nuestra investigación que el alto Síndrome de Burnout se da por igual en enfermeros varones y mujeres, constituyendo el estrés por igual para ambos géneros. Al respecto el Observatorio Permanente de Riesgos Psicosociales (Comisión Ejecutiva Confederal de UGT, 2006) refiere que es incierto el factor sexo y/o género para la prevalencia de este síndrome. Los estudios al respecto no son todavía suficientemente concluyentes y precisan de una mayor atención y mayor análisis.

\section{Declaración de financiamiento y de conflicto de intereses:}

El estudio fue financiado por la autora, quien declara no tener algún tipo de conflicto de interés en la investigación realizada.

\section{Correspondencia}

Yrma Nilda Broncano Vargas

Hospital Nacional Docente Madre Niño "San Bartolomé"

Av. Alfonso Ugarte 825 Lima - Teléfono 2010400

Correo electrónico: yrma.nilda@hotmail.com 


\section{REFERENCIAS BIBLIOGRÁFICAS}

Albaladejo, R., Villanueva, R., Ortega P., Astasio, P., Calle, M. y Domínguez, V. (2004). Síndrome de Burnout en el personal de enfermería de un hospital de Madrid. Rev. Esp. Salud Pública, 78(4), 505-516.

Arce, V., Masgo, L. y Vásquez C. (2006). Síndrome de Burnout en profesionales de enfermería de la Unidad de Cuidados Críticos del Hospital Guillermo Almenara Irigoyen. Revista de Ciencias de la Salud, 1(1), 28.

Arias, F. (2008). Síndrome del desgaste profesional o de Burnout en el personal de enfermería. Rev. Enfermería Actual en Costa Rica. Recuperado de: http://www. revenf.ucr.ac.cr/desgaste.pdf> ISSN 1409-4568

Ben, R., Bauzá, V., Cruz, S. y Calvo M. (2009). "Caracterización gnoseológica sobre el comportamiento organizacional y la satisfacción laboral" en Contribuciones a la Economía. Recuperado de: http:// www.eumed.net/ce/2009a/

Cantera, J. (2008). Evaluación de la satisfacción laboral: métodos directos e indirectos. Instituto Nacional de Seguridad e Higiene en el Trabajo. España: SIAFA. Recuperado de: http://www.insht.es/InshtWeb/Contenidos/ Documentacion/FichasTecnicas/NTP/Ficheros/201a300/ ntp_212.pdf

Colegio de enfermeras(os) del Perú. Consejo Nacional. (2008) "Normas de gestión de la calidad del cuidado enfermero” Lima - Perú.

Colunga, C., Domínguez, R., González, M., Del Castillo, G. y Solorio, S. (2008). "Satisfacción laboral y Burnout en enfermeras de tercer nivel de atención en Guadalajara". 2 do Foro de las Américas en investigación sobre factores psicosociales. Estrés y salud mental en el trabajo.

Comisión Ejecutiva Confederal de UGT (2006). Guía sobre el síndrome de quemado (Burnout). Observatorio permanente riesgos psicosociales. Madrid: Fundación para la prevención de riesgos laborales. Recuperado de:http://extranet.ugt.org/saludlaboral/OPRP/ Publicaciones/Guas/Gu\%C3\%ADas\%20Generales/ Gu\%C3\%ADa_Burnout.pdf.

Díaz, M., Stimolo, M. y Caro, N. (2010) Satisfacción laboral y síndrome de desgaste laboral en enfermeros de hospitales públicos Córdoba-Argentina. Med. segur. Trab., 56(218), 22-38 Recuperado de: http://scielo. isciii.es/scielo.php?script=sci_arttext\&pid=S0465546X2010000100003\&lng=es.http://dx.doi. org/10.4321/S0465-546X2010000100003.

Dolan, N. (1987). The relationship between burnout and job satisfaction in nurses. $J$ Adv Nurs (12) 3, 12 .

Chiang, M., Salazar, C. y Núñez, A. (2007). Clima organizacional y satisfacción laboral en un establecimiento de salud estatal. Theoria; 16(2) $61-76$.

Chiang, M., Salazar, M., Huerta, C., Nuñez, A. (2008). Clima organizacional y satisfacción laboral en organizaciones del sector estatal. Universum. 23(2), 66-85.
Farfán, M. (2006). Construcción, validez y confiabilidad de un instrumento para medir satisfacción laboral en mujeres. (Tesis doctoral). Universidad Iberoamericana, México.

Freudenberger, H, (1974). Staff burnout, Journal of Social Issues; (30) 159-165.

Gil-monte, J., García-Juesas, E., Núñez, N., Carretero, M., Roldán, M. y Caro (2006). Validez factorial del Cuestionario para la Evaluación del Sindrome de Quemarse por el Trabajo (CESQT). 10(3), 1-3.

Gil-monte, P. y Marucco, M. (2008). Prevalencia del "síndrome de quemarse por el trabajo" (Burnout) en pediatras de hospitales generales. Rev. Saúde Pública, 42(3), 450-456

Gil-monte, P. (2009). Algunas razones para considerar los riesgos psicosociales en el trabajo y sus consecuencias en la salud pública. Madrid. Rev. Esp. Salud Pública, 83(2), recuperado de: http://scielo. isciii.es/scielo.php? script $=$ sci pdf\&pid $=\mathrm{S} 1135$ $57272009000200003 \& \operatorname{lng}=$ es\&nrm=iso\&tlng=es

Gil-monte, P. (2011). Cuestionario para la evaluación del síndrome de quemarse por el trabajo. Madrid: TEA Ediciones.

González, A., Oramas, A., Alvarez, S., Oliva, E. (2006). El Síndrome de Burnout. Un desafío ético para alcanzar la excelencia en enfermería. Revista Habanera de Ciencias Médicas, 5(4), 1-5.

Hernández, V., Quintana, L., Mederos R., Guedes, R. y García, N. (2009). Motivación, satisfacción laboral, liderazgo y su relación con la calidad del servicio. Rev. Cub. Med. Mil. 38 (1), 2-8.

López, Zegarra y Cuba. (2006). Factores asociados al Síndrome de Burnout en enfermeras de Emergencia del Hospital Nacional Guillermo Almenara Irigoyen. Lima. Revista de Ciencias de la Salud. 1(1), 53.

Martínez-López, C. y López-Solache, G. (2005) Características del Síndrome de Burnout en un grupo de enfermeras mexicanas. Archivos en Medicina Familiar, 7(1), 6-9.

Marucco Mariana, Gil-monte Pedro R. Satisfacción laboral en pediatras del conourbano bonaerense, Región Sur. Rev. Chilena Pediatría. 2007; vol 78 (n.5): 489-493.

Maslach, C., Jackson, SE. (1981). MBI: Maslach Burnout Inventory. Manual. Palo Alto: University of California, Consulting Psychologists Press.

Milachay, D. (2006). Factores personales y organizacionales relacionadas con el Síndrome de Burnout del profesional de enfermería del Hospital Rene Toche Groppo. EsSalud Chincha. (Tesis de Maestría). Universidad San Martin de Porres. Perú.

Morales, C. (2006). Nivel de estrés laboral de las enfermeras que laboran en las áreas críticas del Instituto Nacional 
de Salud del Niño. (Tesis de Licenciatura). Universidad Nacional Mayor de San Marcos. Lima.

Olivares, V. E y Gil -Monte, P. R. (2007) Análisis de las propiedades psicométricas del "Cuestionario para la Evaluación del Sindrome de Quemarse por el Trabajo" (CESQT) en profesionales chilenos. Ansiedad y Estrés. (13) 229-240.

Ortega, R. y López, R. (2004). El burnout o síndrome de estar quemado en los profesionales sanitarios: revisión y perspectivas. International. Journal of Clinical and Health Psychology, 4(1), 137-160.

Ozturk, H., Bahcecik, N. \& Baumann, S. L. (2006). Nursing satisfaction and job enrichment in Turkey. Nurs Sci $Q$, 19(4) 360-365.

Paredes, R. (2006). Relación entre el estrés ocupacional y la satisfacción laboral en el personal de enfermería que labora en el servicio de emergencia pediátrica y de adultos del hospital "Dr. Pedro Emilio Carrillo". (Tesis especialidad). Universidad Católica Andres Bello, Venezuela.

Rios, R. y Godoy, C. (2008). Satisfacción laboral en un colectivo de enfermería de urgencias. España. Revista cientifica de la sociedad española de enfermería de urgencias y emergencias. (0), 1-6.

Ríos-Risquez y Godoy-Fernández, C. (2008). Relación entre satisfacción laboral y salud general percibida en profesionales de enfermería de urgencias. Rev. España Enferm. Clin., 18 (3), 134-41.
Santamaría, N. (2010). Acciones o estrategias individuales, grupales e institucionales para manejar el estrés laboral de las enfermeras de áreas y servicios de oncología. El portal de la salud. Recuperado de: http://www. elportaldelasalud.com/index.php?option $=\mathrm{com}_{-}$ content\&task $=$ view\&id $=239 \&$ Itemid $=155$

Sociedad Española de Cuidados Paliativos. (2008) Guía de prevención de BURNOUT para profesionales de cuidados paliativos. España: Aran Ediciones S.L.

Tello, J. (2010). Nivel de Síndrome de Burnout y estrategias de afrontamiento en enfermeros de los servicios críticos del Hospital Nacional Daniel Alcides Carrión. (Tesis de Maestría) Universidad Nacional Mayor de San Marcos. Lima.

Thomaé, M., Ayala, E., Sphan, M., Stortti, M. (2006). Etiología y prevención del Síndrome de Burnout en los trabajadores de la salud. Revista de Posgrado de la VI Cátedra de Medicina. (153), 18-21.

Tuesca-Molina, R., Iguarán, M., Suárez, M., Vargas, G. y Vergara, D. (2006). Síndrome de desgaste profesional en enfermeras/os del área metropolitana de Barranquilla. Salud Uninorte, 22 (2), 84-91.

Recibido: 08/05/2014 Aceptado: 17/10/2014 